\title{
Correction to: Unsupervised and scalable subsequence anomaly detection in large data series
}

\author{
Paul Boniol $^{1}$ (1) $\cdot$ Michele Linardi $^{2} \cdot$ Federico Roncallo $^{2} \cdot$ Themis Palpanas $^{2} \cdot$ Mohammed Meftah $^{1} \cdot$ Emmanuel Remy $^{1}$
}

Published online: 31 August 2021

๑) Springer-Verlag GmbH Germany, part of Springer Nature 2021

\section{Correction to: The VLDB Journal}

https://doi.org/10.1007/s00778-021-00655-8

There are errors in the original publication.

In the Sect. 5.7:

- 3rd line: "NYC Marathon" corrected as "Daylight Saving Time (DST)".

- 9th line: "and the bad weather of January 18, 2015 (g)" corrected as "and the bad weather of January 18, followed by the Martin Luther King (MLK) day (January 19)".

In the Sect. 2.2:

1st sentence of 2 nd paragraph: "Nevertheless, we claim that the way discords are defined cannot lead to solutions that are useful in practice." corrected as "Nevertheless, we claim that the way discords are defined may in some situations complicate the discovery of anomalies."

The original article has been corrected.

The original article can be found online at https://doi.org/10.1007/ s00778-021-00655-8.

Paul Boniol

Paul.Boniol@edf.fr

Michele Linardi

Michele.Linardi@parisdescartes.fr

Federico Roncallo

Federico.Roncallo@parisdescartes.fr

Themis Palpanas

Themis.Palpanas@parisdescartes.fr

Mohammed Meftah

Mohammed.Meftah@edf.fr

Emmanuel Remy

Emmanuel.Remy@edf.fr

EDF R\&D, Paris, France

2 Université de Paris, Paris, France
Publisher's Note Springer Nature remains neutral with regard to jurisdictional claims in published maps and institutional affiliations. 